\title{
Early life factors in relation to albuminuria and estimated glomerular filtration rate based on cystatin $C$ and creatinine in adults from a Swedish population-based cohort study
}

\author{
Agne Laucyte-Cibulskiene ${ }^{1,2}$ (D) . Shantanu Sharma ${ }^{1} \cdot$ Anders Christensson $^{1,2} \cdot$ Peter M. Nilsson $^{1}$
}

Received: 27 April 2021 / Accepted: 17 September 2021 / Published online: 8 October 2021

(c) The Author(s) 2021

\begin{abstract}
Background Early life factors influence the number of nephrons a person starts life with and a consequence of that is believed to be premature kidney ageing. Thus, we aimed to identify early life factors associated with cystatin $\mathrm{C}$ and creatinine-based estimated glomerular filtration (eGFR) rate equations and urine -albumin-to-creatinine ratio after a follow-up of 46-67 years. Methods The study included 593 Swedish subjects without diabetes mellitus from the Malmo Diet Cancer Cohort. Perinatal data records including birth weight, gestational age, placenta weight and maternal related risk factors were analysed. eGFR was determined by Chronic Kidney Disease Epidemiology (CKD-EPI), the Lund-Malmö revised and Caucasian, Asian, Paediatric, and Adult (CAPA) equations. Postnatal growth phenotypes were defined as low $(\leq 0)$ or high $(>0)$ birth weight $\mathrm{z}$-score, or low ( $\leq$ median) or high ( $>$ median) body mass index at 20 years of age.

Results In women, lower birth weight was associated with lower eGFR (CAPA; CKD-EPI cystatin C). Birth weight z-score predicted adult albuminuria specifically in men (OR 0.75, 95\% CI $[0.58 ; 0.96])$. Women with high birth weight z-score and low BMI at 20 years had lower eGFR (CAPA; CKD-EPI cystatin C; $p=0.04$ ). Men with high birth weight $\mathrm{z}$-score and high BMI at 20 years had lower risk for albuminuria (OR 0.35, 95\% CI [0.12; 0.93]).

Conclusions Lower birth weight, prematurity and postnatal growth curve have a potential sex- specific effect of early exposure to an adverse environment on lower cystatin C-based eGFR and albuminuria later in life. Cystatin C compared to creatinine -eGFR equations shows a higher ability to detect these findings.
\end{abstract}

Agne Laucyte-Cibulskiene

agne.laucyte-cibulskiene@med.lu.se

1 Department of Clinical Sciences, Skane University Hospital, Lund University, Ruth Lundskogs gata 14, 20502 Malmö, Sweden

2 Department of Nephrology, Skane University Hospital, Lund University, 20502 Malmö, Sweden 


\section{Graphic abstract}

Early life factors in relation to albuminuria and estimated glomerular filtration rate based on cystatin $\mathrm{C}$ and creatinine in adults from a Swedish population-based cohort study

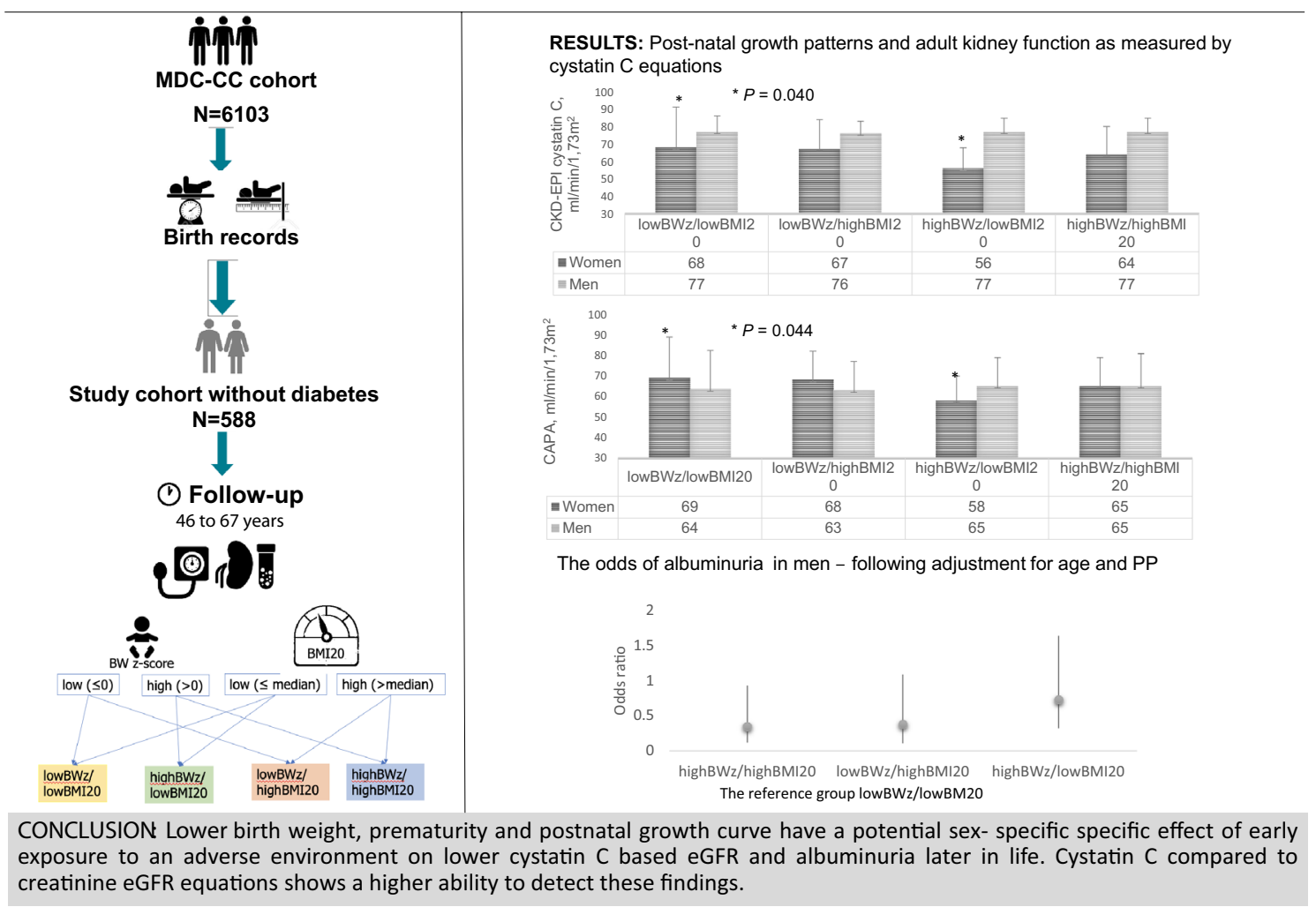

Keywords Albuminuria $\cdot$ Birth weight $\cdot$ Cystatin C $\cdot$ Early life $\cdot$ Epidemiology $\cdot$ Estimated glomerular filtration rate

\section{Introduction}

\section{Background}

Kidney senescence, dependent on predicted anatomical and structural changes, is a separate process from kidney disease [1] and manifests with declining nephron number and size. Aged-induced glomerulosclerosis is followed by compensatory glomerular hypertrophy, glomerular basement membrane (GBM) thickening, tubular atrophy, interstitial fibrosis, and arteriosclerosis [2]. Normal kidney ageing and chronic kidney disease (CKD) share similar pathophysiological [2] and maladaptive repair [3] pathways despite different causality. CKD is very common in the general population and as many as $10 \%$ of individuals suffer from this neglected "non-communicable disease" associated with higher mortality [4]. Among CKD risk factors, e.g. ageing, gender, family history, obesity, smoking, hypertension, diabetes mellitus, etc. [5], early life factors also play an important role in CKD progression and renal outcome prediction $[6,7]$.
Renal functional capacity is influenced by factors acting early in life, such as intrauterine environment, maturity, birth weight, length at birth, placental weight, etc. [7-9]. Early life factors influence the number of nephrons a person starts with at birth, and the consequence of low nephron number is believed to be premature kidney ageing, hypertension and CKD later in one's lifetime [10]. There is a debate on whether low birth weight newborns have low podocyte endowment [11] which increases the risk of renal pathology. In utero perturbation leads to altered kidney development that affects low birth weight and/or diminished nephron number causing decreased filtration surface area/altered sodium handling and eventually leading to increased programmed risk of adult hypertension [12] and kidney diseases [13].

In a recent meta-analysis [7] the strong relationship between low birth weight, or small for gestational age, and renal dysfunction in adulthood was observed. Another longitudinal result came from the Helsinki Birth Cohort study [8] which found that smaller body size at birth influences the onset of CKD, although mainly in men. There are other 
definitive triggers for the development of CKD such as prematurity [9] and preeclampsia [14]. On the contrary, the role of both high birth weight, a proven risk marker for the metabolic syndrome, and maternal obesity [15] has to be elucidated for kidney health.

The evaluation of kidney function is based on glomerular filtration rate (GFR) that can be measured (mGFR) or estimated (eGFR) from equations based on creatinine or cystatin $\mathrm{C}$ which are used worldwide [16]. During previous decades it was shown that cystatin $C$ is superior to creatinine in estimating kidney function [17]. Notably, most reports addressing early life factors in the context of adult kidney function use creatinine-based eGFR equations and/or albuminuria but lack longer follow-up ( $>30$ years) $[7,18]$.

\section{Objectives}

We aimed to identify how early life factors affect adult kidney function, by introducing cystatin C-based GFR equations and kidney damage, determined by urine albuminto-creatinine ratio (UACR), in a cohort with very long follow-up, i.e., more than 40 years. We hypothesize that in middle-aged and elderly Swedish subjects, changes in kidney function capacity, represented as lower eGFR, and albuminuria, are influenced by early life factors such as low birth weight, prematurity, discrepant birth weight to placental weight ratio, and postnatal growth mismatch phenotype.

\section{Methods}

\section{Study design and settings}

The observational cohort study was performed with subjects that participated in the population-based Malmö Diet and Cancer Study (MDCS) [19] between 1991 and 1996. MDCS comprises 17,035 women and 11,063 men born between 1923 and 1950 and residing in Malmö. Furthermore, a random $50 \%$ of MDCS participants were invited to participate in carotid artery disease epidemiological analysis (the cardiovascular arm of MDCS (MDCS-CC)). Due to lower response rate a total of 6,103 participants completed the MDCS-CC study [20, 21].

\section{Participants}

In this study, MDCS-CC participants without diabetes mellitus for whom perinatal data records were available including birth weight, birth length, head circumference, gestational age, placenta weight, and maternal risk factors at baseline (year 1926-1945), and cystatin C and creatinine measurements after the mean follow-up time of $58 \pm 5$ years (range: 46 to 67 years) (96 women and 497 men) were included. For more detailed participant recruitment and data collection see Gerdtsson et al. [22] and Lahmann et al. [23]. The mean follow-up time was $58 \pm 5$ years (range $46-67$ years).

\section{Measurements}

\section{Early life factors}

Birth records were collected from archives at local and regional hospitals and were located using methods as described elsewhere [22, 23]. Birth records include data regarding birth weight (grams), length at birth (centimetres), head circumference (centimetres), placental weight (grams), and last menstrual period (day). Gestational age at delivery was calculated from last menstruation period until the birth week, prematurity was defined as being born alive before 37 weeks of pregnancy. Birth weight to placenta weight ratio was calculated as birth weight $(\mathrm{g}) /$ placenta weight $(\mathrm{g})$.

\section{Postnatal growth phenotype}

The mismatch term defines pre- and postnatal growth patterns influencing body weight at 20 years of age. To test different postnatal growth curves, or mismatch phenotypes, we divided all participants into four subgroups by combining sex-specific low $(\leq 0)$ or high $(>0)$ birth weight $\mathrm{z}$-score (low-birth weight-z-score or high-birth weight-z-score, respectively) with low ( $\leq$ median) or high ( $>$ median) recalled body mass index (BMI) at 20 years of age (lowBMI20 or highBMI20, respectively). These four subgroups were as follows: low-birth weight-z-score/low BMI20, low-birth weight-z-score/high BMI20 (the mismatch or postnatal catch-up growth phenotype), high-birth weight-z-score/low BMI20, and high-birth weight-z-scorel high BMI20.

Birth weight $\mathrm{z}$-scores (gender-specific birth weight z-score and combined birth weight z-score) were acquired by using equation from official Swedish growth-charts as reported by Marsal et al. [24]. BMI20 calculation: selfreported body weight $(\mathrm{kg})$ at 20 years of age divided by squared height $(\mathrm{m})$.

\section{Follow-up measurements}

All participants underwent anthropometric measurements (weight (kilograms), height (centimetres)) and systolic and diastolic blood pressure (SBP and DBP, $\mathrm{mmHg}$ ) measurements obtained by trained nurses between 1991 and 1996 . Pulse pressure (PP) was calculated as follows: SBP-DBP $(\mathrm{mmHg})$. Questions concerning self-reported body weight at 20 years of age were included in a self-administrated questionnaire [25]. Fasting blood samples were drawn and immediately frozen to $-80{ }^{\circ} \mathrm{C}$ and stored in a biobank as 
reported elsewhere [26]. Plasma creatinine $(\mu \mathrm{mol} / \mathrm{L})$ concentration was analysed by the Jaffé method [27] and using a calibrator traceable to the international standardization with isotope dilution mass spectrometry (IDMS) [28]. Plasma cystatin C (mg/L) was measured with a particleenhanced immunonephelometric assay (N Latex Cystatin; Dade Behring, Deerfield, IL, USA). The values for cystatin $\mathrm{C}$ were analysed before the introduction of the world calibrator in 2010 and thus not standardized [29]. The reference values for the method were $0.53-0.95 \mathrm{mg} / \mathrm{L}$. In order to standardize cystatin $\mathrm{C}$ values, we used cystatin $\mathrm{C}$ and creatinine within 2.5-97.5th percentile in a reference Swedish cohort and values in the same range in MDC-CC. Furthermore, sex-divided linear regression models with $\ln$ (cystatin c) as the dependent variable adjusted for age and for $\ln$ (creatinine) were created both in the reference cohort and in MDC-CC, and predicted cystatin $\mathrm{C}$ concentrations were acquired. Corrected cystatin $\mathrm{C}$ values were calculated by using the formula below:
Continuous variables are expressed as mean \pm standard deviations (SD) in parentheses, discrete variables as medians with min-max values in parentheses, and categorical variables as percentages with numbers in parentheses. Where appropriate, the equality of two populations for normally distributed continuous data were tested by an $F$ test, and only after this did we perform a Student's $t$ test. Nonparametric tests, such as a two-sample Wilcoxon test, was performed on not normally distributed data. A Chi-square test was applied to categorical variables. To determine the correlation between two variables, a Pearson correlation test was performed and depicted as scatterplots. The ANOVA test was applied for comparison of age-adjusted eGFR equations in different postnatal growth phenotypes.

Multivariable linear regression analyses with creatinine and cystatin $\mathrm{C}$ equations as dependent variables were performed to identify early life factors independently associated with kidney function. UACR was categorized in two groups: normal UACR if $<3 \mathrm{mg} / \mathrm{mmol}$, albuminuria if $\geqslant 3 \mathrm{mg} / \mathrm{mmol}$.

Corrected Cys $C=C y s C_{M D S-C C}+$ predicted Cys $C_{\text {reference }}-$ predicted $C y s C_{M D S-C C}$

Cys C-cystatin C, reference-the reference cohort.

\section{Kidney function evaluation}

Estimated glomerular filtration rate was calculated according to 4 different equations: Chronic Kidney Disease Epidemiology Collaboration (CKD-EPI) 2012 creatinine and cystatin C equation [30] (CKD-EPI creatinine, CKD-EPI cystatin C), cystatin C eGFR equation based on Caucasian, Asian, Paediatric, and Adult cohorts [31], the Lund-Malmö revised creatinine based eGFR equation (LMrev) [32].

Urine albumin-to-creatinine ratio $(\mathrm{mg} / \mathrm{mmol})$ was measured in morning urine samples as reported elsewhere [33] and albuminuria was defined as UACR $\geqslant 3 \mathrm{mg} / \mathrm{mmol}$. it is worth noting that $11 \%(n=65)$ of participants had microalbuminuria and $2 \%(n=11)$ had macroalbuminuria.

\section{Ethics}

Each investigator committed to comply with legislation and to conduct the study in line with regulations, in accordance with the 1975 Declaration of Helsinki and its later amendments. The following ethical permissions apply for the cohort: MDCS (LU 51/90, 532/2006).

\section{Statistical analysis}

All statistical analyses were conducted with Rcrmdr version R 3.6.2 GUI 1.70 El Capitan build macOS.
Logistic regression analysis identified early life related variables as risk factors for albuminuria. A $p$ value less than 0.05 was considered significant.

\section{Results}

\section{Participants}

The study sample selection flow chart is presented in Fig. 1.

\section{General characteristics of the study population}

General characteristics for men and women including the early life variables at birth and variables evaluated after a follow-up of 46-67 years are presented in Table 1. eGFR calculated by the CAPA equation and UACR were similar in both sexes. Birth weight percentiles $(\mathrm{P})$ were as follows: in women $\mathrm{P}_{2}-2,408 \mathrm{~g}, \mathrm{P}_{5}-2,550 \mathrm{~g}, \mathrm{P}_{10}-2,747.5 \mathrm{~g}$, $\mathrm{P}_{25}-3,045 \mathrm{~g}, \mathrm{P}_{50}-3,410 \mathrm{~g}, \mathrm{P}_{75}-3,762.5 \mathrm{~g}, \mathrm{P}_{90}-4,055 \mathrm{~g}$, $\mathrm{P}_{95}-4,388.75 \mathrm{~g}, \mathrm{P}_{98}-4,561 \mathrm{~g}$; in men $\mathrm{P}_{2}-2,233.6 \mathrm{~g}$, $\mathrm{P}_{5}-2,650 \mathrm{~g}, \mathrm{P}_{10}-2,850 \mathrm{~g}, \mathrm{P}_{25}-3,200 \mathrm{~g}, \mathrm{P}_{50}-3,550 \mathrm{~g}$, $\mathrm{P}_{75}-3,900 \mathrm{~g}, \mathrm{P}_{90}-4,200 \mathrm{~g}, \mathrm{P}_{95} 4,352 \mathrm{~g}, \mathrm{P}_{98}-4,630.4 \mathrm{~g}$.

\section{Early life factors and adult kidney function}

Birth weight in women was positively correlated with adult kidney function measured by both cystatin $\mathrm{C}$ equations (CAPA and CKD-EPI cystatin C) (Fig. 2). However, there was no association between birth weight and adult kidney 
Fig.1 Flow chart of study sample. $M D C S$ Malmo Diet Cancer study, MDCS-CC Malmo Diet Cancer study Cardiovascular Cohort

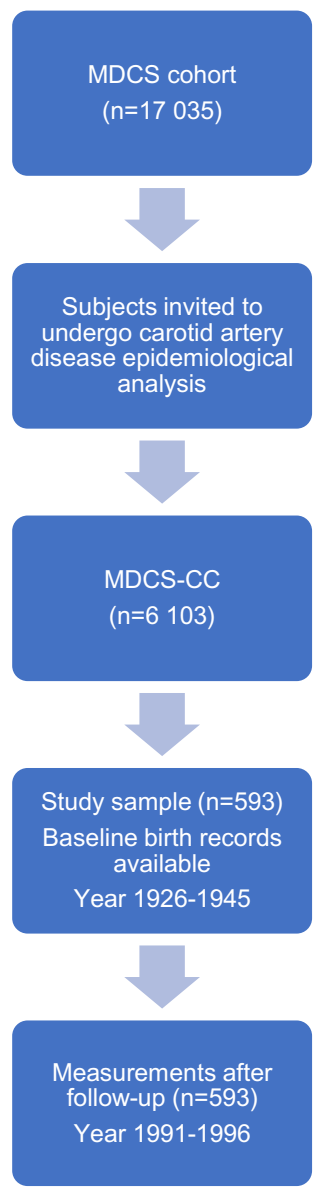

MDCS - Malmo Diet Cancer study; MDCS-CC - Malmo Diet Cancer study Cardiovascular Cohort.

function in men for either cystatin $\mathrm{C}$ or creatinine equations. The correlations between early life factors, e.g. lower birth weight, and elevated UACR observed in the whole cohort were not sex-specific (Fig. 3).

\section{Linear regression analysis with eGFR as dependent variable}

Multivariable linear regression analysis (Table 2) showed that for every $100 \mathrm{~g}$ increase in birth weight (adjusted for gestational age and sex), the predicted eGFR increased by $0.3 \mathrm{~mL} / \mathrm{min} / 1.73 \mathrm{~m}^{2}$ (CKD-EPI cystatin $\mathrm{C}$ equation). The presence of prematurity is associated with predicted eGFR decrease by $3.23 \mathrm{~mL} / \mathrm{min} / 1.73 \mathrm{~m}^{2}$ (CKD-EPI cystatin C equation) over the mean follow-up time of $58 \pm 5$ years. Sexspecific analysis revealed that in women, for every $100 \mathrm{~g}$ increase in birth weight, the predicted eGFR increased by $1.00 \mathrm{~mL} / \mathrm{min} / 1.73 \mathrm{~m}^{2}$ (both CAPA and CKD-EPI cystatin $\mathrm{C}$ equations). Whilst in men, for every increase in 1 unit in birth weight to placenta weight ratio, the predicted eGFR decreased by $1.67 \mathrm{~mL} / \mathrm{min} / 1.73 \mathrm{~m}^{2}$ (LMrev equation).
eGFR in this analysis was adjusted for age and pulse pressure (Table 2). The results of linear regression are visualized in Fig. 4.

\section{Risk of albuminuria}

Logistic regression analysis showed that odds of albuminuria (adjusted for age and pulse pressure) was significantly lower in the subjects with higher either gender-specific or combined birth weight z-score (OR 0.78, 95\% CI [0.61; $0.98]$ ). This observation was specific for men (OR $0.75,95 \%$ CI $[0.58 ; 0.96])$.

\section{Growth mismatch subgroup comparison: sex-specific differences}

We found that women with high-birth weight-z-scorel lowBMI20 phenotype compared to low-birth weight-z-scorel lowBMI20 phenotype (Fig. 5) had significantly lower eGFR adjusted for age and pulse pressure $(p=0.044$ for CAPA, 
Table 1 Study populationclinical characteristics and descriptive analyses

\begin{tabular}{lllr}
\hline & Women $(n=96)$ & Men $(n=497)$ & $P$ \\
\hline Age, years & $56(5)$ & $58(5)$ & $<0.001$ \\
Body weight, kg & $70(13)$ & $82(12)$ & $<0.001$ \\
Height, cm & $165(6)$ & $177(7)$ & $<0.001$ \\
Body mass index, kg/m ${ }^{2}$ & $25.6(4.4)$ & $26.1(3.3)$ & 0.343 \\
Systolic blood pressure, mmHg & $136(18)$ & $144(18)$ & $<0.001$ \\
Diastolic blood pressure, mmHg & $84(9)$ & $89(9)$ & $<0.001$ \\
Pulse pressure, mmHg & $53(14)$ & $55(14)$ & 0.096 \\
Gestational age, weeks & $39(2)$ & $40(2)$ & 0.052 \\
Head circumference, cm & $35(2)$ & $35(2)$ & 0.965 \\
Birth weight, g & $3,396(570)$ & $3,534(545)$ & 0.032 \\
Placenta weight, g & $624(124)$ & $631(138)$ & 0.716 \\
Birth weight to placenta weight ratio & $5.5(0.8)$ & $5.7(1.0)$ & 0.070 \\
Expected weight [23] (adjusted for gestational age), g & $3,477(374)$ & $3,550(392)$ & 0.090 \\
Expected weight [23] (adjusted for gestational age, sex), g & $3,417(354)$ & $3,606(415)$ & $<0.001$ \\
Cystatin C, mg/L & $1.2(0.2)$ & $1.2(0.2)$ & 0.684 \\
Creatinine, $\mu$ mol/L & $80(12)$ & $93(14)$ & $<0.001$ \\
CAPA [31] & $64(16)$ & $65(16)$ & 0.858 \\
LMrev [32] & $81(12)$ & $70(11)$ & $<0.001$ \\
CKD-EPI cystatin C [30] & $63(17)$ & $77(8)$ & $<0.001$ \\
CKD-EPI creatinine [30] & $73(13)$ & $79(13)$ & $<0.001$ \\
Urine albumin-to-creatinine ratio, mg/mmol & $2.1(5.4)$ & $3.1(14.3)$ & 0.504 \\
\hline
\end{tabular}

Data expressed as Mean $( \pm \mathrm{SD})$, and statistical comparisons by Student's $t$ test

eGFR estimated glomerular filtration rate, CAPAcystatin C eGFR equation based on Caucasian, Asian, Paediatric, and Adult cohorts, LMrev the Lund-Malmö revised creatinine based eGFR equation, CKD-EPI cystatin $C$ the Chronic Kidney Disease Epidemiology Collaboration cystatin C equation, CKD-EPI creatinine the Chronic Kidney Disease Epidemiology Collaboration creatinine equation and $p=0.040$ for CKD-EPI cystatin C). These associations were absent in men.

The odds of albuminuria (adjusted for age and pulse pressure) were significantly lower in men with high-birth weight-z-score/highBMI20 phenotype compared to the reference group with low-birth weight-z-score/lowBMI20 (OR $0.35,95 \%$ CI $[0.12 ; 0.93])$. The odds of albuminuria in other subgroups in men were as following: low-birth weight-zscore/highBMI20_OR 0.38, 95\% CI [0.11; 1.09], high-birth weight-z-score/lowBMI20_OR 0.73, 95\% CI [0.33; 1.64].

\section{Discussion}

This observational, population-based study supports our hypothesis that in middle-aged and elderly Swedish subjects sex-specific associations exist between early life factors and kidney function, defined as cystatin C-based eGFR, as well as with albuminuria. This study also confirms that cystatin $\mathrm{C}$ compared to creatinine is a more sensitive marker for demonstrating these associations.

Despite the growing evidence that low birth weight together with developmental exposure to an adverse environment acting on the kidney might result in lower nephron/ podocyte endowment $[11,34]$, there is still a critical need to identify early life factors that affect kidney function during the life course with the reporting of sex-stratified analyses.

Previous studies, including those listed in two recent meta-analyses, [7, 35] have mainly used creatinine-based equations for calculating adult eGFR with few exceptions of measured GFR [36] in women and cystatin $\mathrm{C}$ equation in a British population [37]. Moreover, the participants in these studies, except for two British reports [37, 38], were younger than 30 years old. Hence, most previous studies were based on plasma creatinine eGFR equations and had a significantly shorter follow-up time compared to our study. We show that subjects with lower birth weight adjusted for gestational age and sex, or those born preterm, are characterised by lower adult eGFR as determined by the CKD-EPI cystatin $\mathrm{C}$ equation, but not by eGFR creatinine equations. In women this association was even more pronounced, revealing a significant association between low birth weight and low eGFR acquired by both cystatin $\mathrm{C}$ equations (CAPA and CKD-EPI cystatin C).

Interestingly, we found that in men a decreased birth weight to placenta weight ratio, reflecting suboptimal fetal growth, when adjusted for age, gestational age, parity and pulse pressure, was related to higher eGFR based 

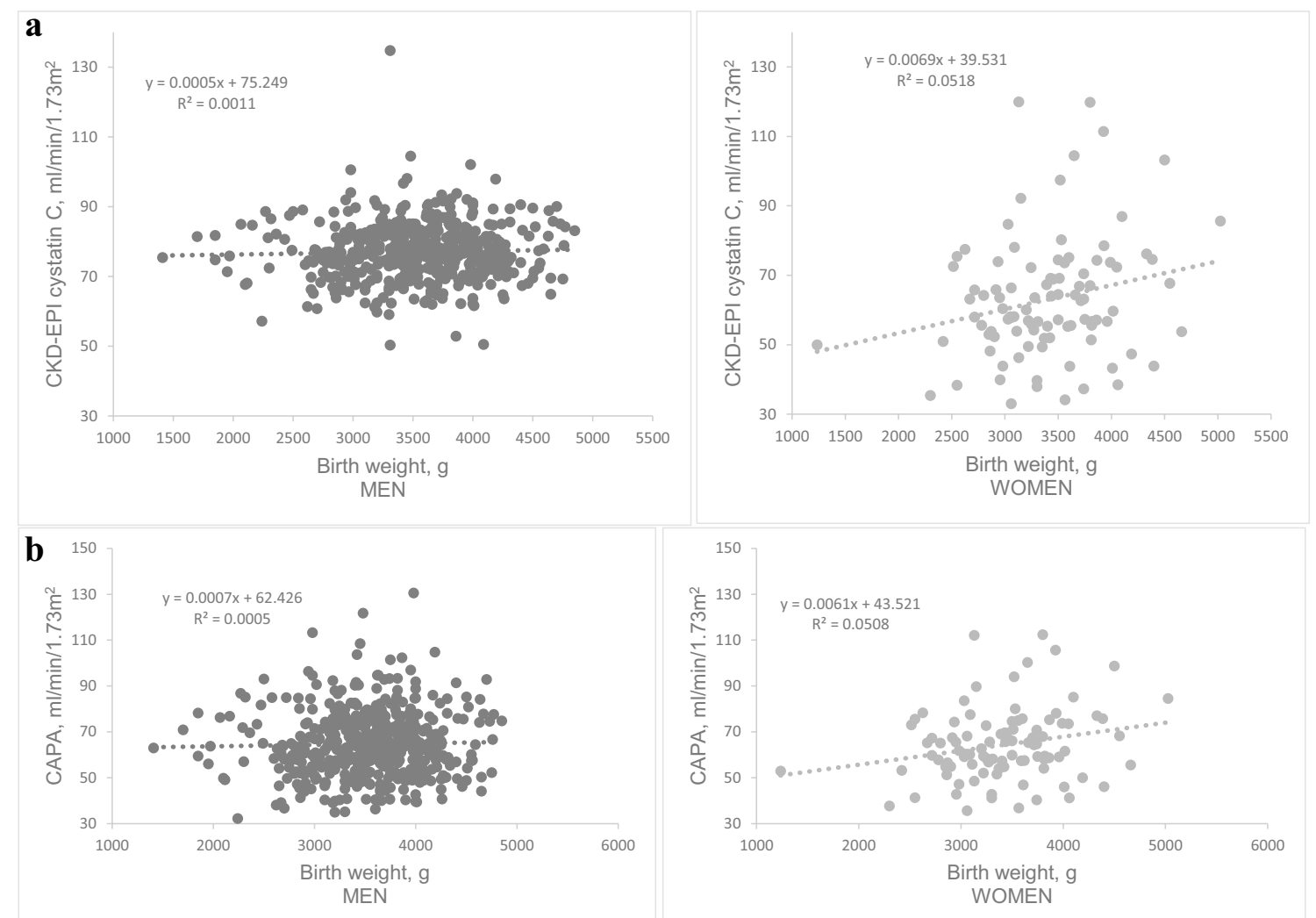

x-axis - birth weight, $\mathbf{y}$ - axis - eGFR measured by using CKD-EPI cystatin C (a) or CAPA (b).

Correlation coefficients: a. women: $\mathrm{r}=0.22, P=0.03$, men: $\mathrm{r}=0.02, P=0.71$; b. women: $\mathrm{r}=0.23, P=0.03$, men: $\mathrm{r}=0.03, P=0.55$.

Fig.2 Birth weight correlation with adult kidney function measured by cystatin $\mathrm{C}$ equations in men and women. $x$ axis-birth weight, $y$ axis-eGFR measured by using CKD-EPI cystatin C (a) or CAPA (b). Correlation coefficients: a women: $r=0.22, P=0.03$, men: $r=0.02, P=0.71$; b women: $r=0.23, P=0.03$, men: $r=0.03$, $P=0.55$
Fig.3 Urine albumin-tocreatinine ratio correlation with birth weight both in men and women. $x$ axis - birth weight, $y$ axis-log urinary albuminto-creatinine ratio (UACR). Correlation coefficient for the whole population $r=-0.131$, $P=0.012$

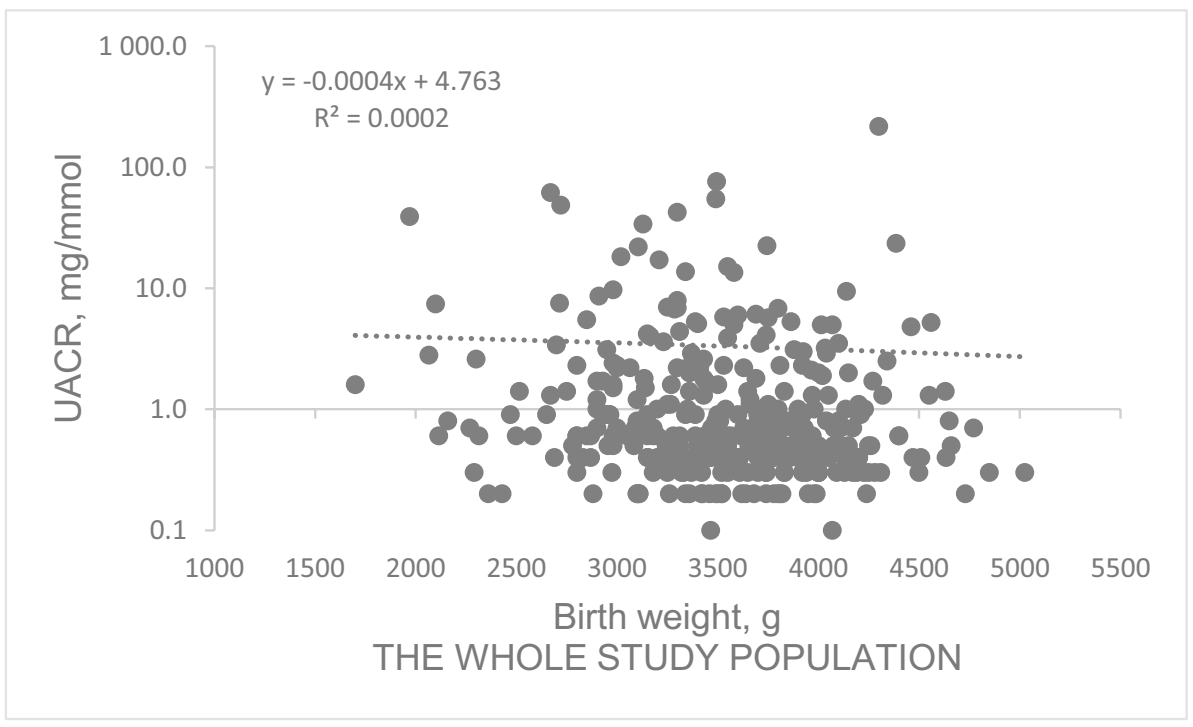

$\mathbf{x}$-axis - birth weight, $\mathbf{y}$-axis - log urinary albumin-to-creatinine ratio (UACR).

Correlation coefficient for the whole population $\mathrm{r}=-0.131, P=0.012$. 
Table 2 Early life factors as determinants of adult kidney function, measured by cystatin C or creatinine eGFR equations-following adjustments for age and pulse pressure

\begin{tabular}{llr}
\hline & $\beta$ & SE \\
\hline All the subjects & CKD-EPI cystatin C & $P$ \\
Birth weight, g & 0.002 & 0.001 \\
Birth weight (adjusted for gestational age, sex), g & 0.003 & 0.001 \\
Prematurity, yes & -3.23 & 0.015 \\
& LMrev & 0.003 \\
Birth weight to placenta weight ratio (adjusted for gestational age, parity) & -1.99 & 0.043 \\
WOMEN & CAPA & 0.015 \\
Birth weight, g & 0.001 & 0.002 \\
& CKD-EPI cystatin C & 0.045 \\
Birth weight, g & 0.001 & 0.003 \\
MEN & LMrev & 0.040 \\
Birth weight to placenta weight ratio (adjusted for gestational age, parity) & -1.67 & 0.89 \\
\hline
\end{tabular}

Multivariable linear regression analysis

Multiple linear regression analysis results are also shown in Fig. 4

CAPA cystatin C eGFR equation based on Caucasian, Asian, Paediatric, and Adult cohorts, LMrev the Lund-Malmö revised creatinine based eGFR equation, CKD-EPI cystatin $C$ the Chronic Kidney Disease Epidemiology Collaboration cystatin $\mathrm{C}$ equation, CKD-EPI creatinine the Chronic Kidney Disease Epidemiology Collaboration creatinine equation

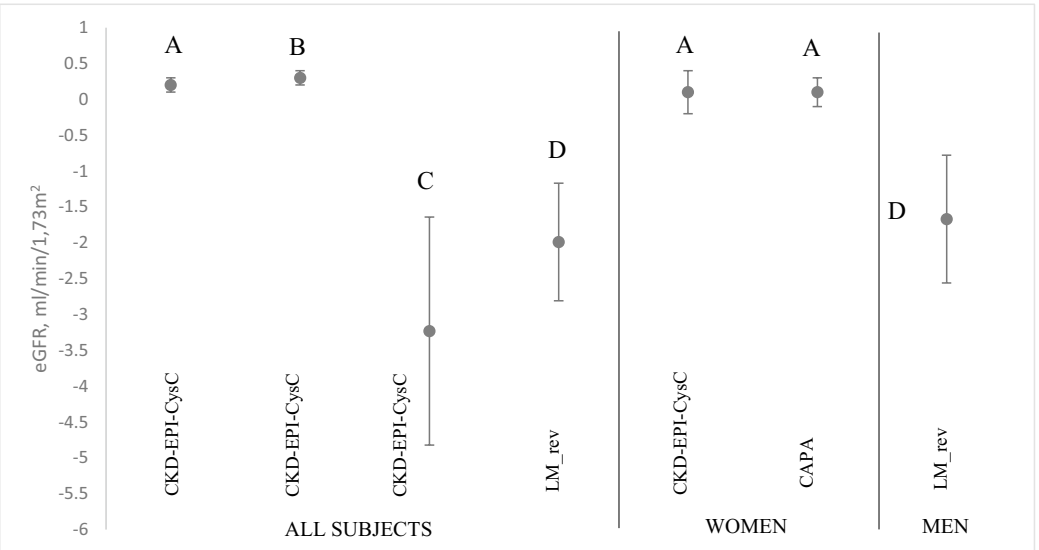

\footnotetext{
This figure shows how birth weight, birth weight to placenta weight ratio unit increase and the presence of prematurity are associated with the predicted eGFR after the mean follow-up time of $58+/-5$ years.

x-axis - early life factors: A - $100 \mathrm{~g}$ increase in birth weight, unadjusted; B - $100 \mathrm{~g}$ increase in birth weight (adjusted for gestational age and sex), C - presence of

prematurity; D - birth weight to placenta weight ratio one unit increase, adjusted for gestational age and parity; $\mathbf{y}$-axis - estimated glomerular filtration ratio.
}

Fig.4 Early life factors as determinants of adult kidney function, measured by cystatin $\mathrm{C}$ or creatinine eGFR equations-following adjustments for age and pulse pressure. Depicting of multiple regression analysis. This figure shows how birth weight, birth weight to placenta weight ratio unit increase and the presence of prematurity are associated with the predicted eGFR after the mean follow-up time

on creatinine (LMrev equation). In contrast, a decreased birth weight to placenta weight ratio has been previously reported to be related to worse perinatal outcome [39] and both neonatal and maternal morbidity [40]. This contradicts data from a Norwegian study [41] that showed an elevated risk for fetal death linked both to small and large of $58 \pm 5$ years. $x$ axis-early life factors: A $100 \mathrm{~g}$ increase in birth weight, unadjusted; B $100 \mathrm{~g}$ increase in birth weight (adjusted for gestational age and sex), $\mathbf{C}$ presence of prematurity; $\mathbf{D}$ birth weight to placenta weight ratio one unit increase, adjusted for gestational age and parity; y axis-estimated glomerular filtration ratio

placentas relative to birth weight in preterm births, and only in the group with small placentas relatively to birth weight at term. We propose that our finding may reveal those (male) individuals with compensatory glomerular hyperfiltration, a marker of both metabolic risk [42] and initial stages of CKD [43]. 
a CKD-EPI cystatin C

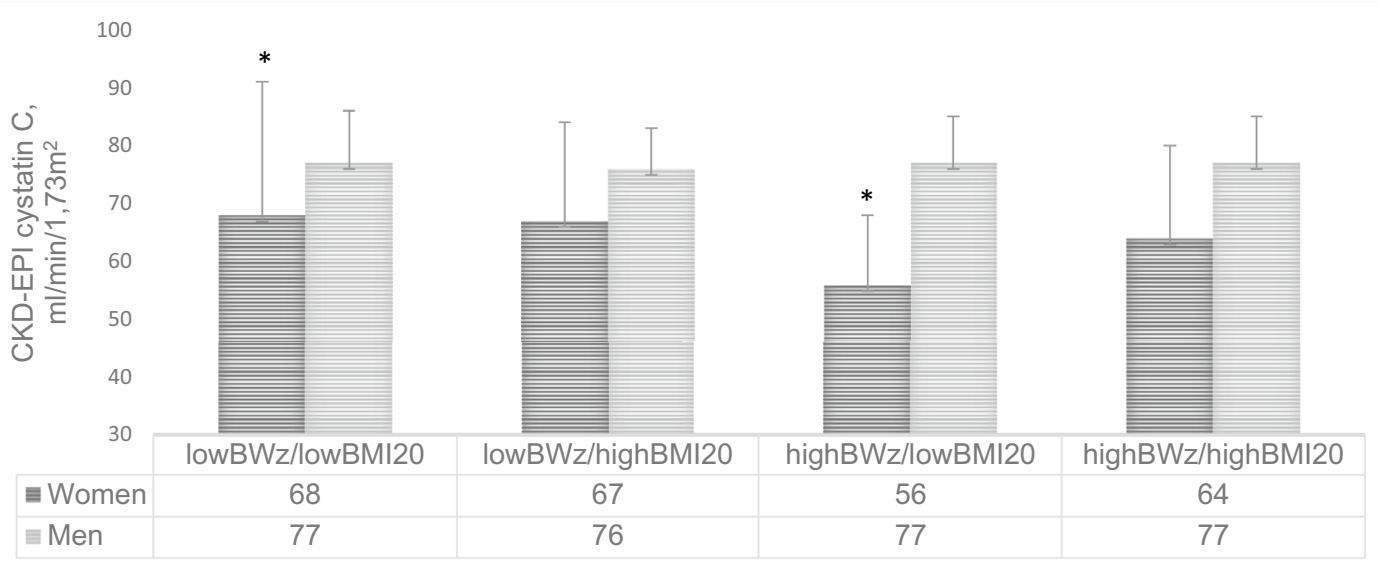

* $P=0.040$

b CAPA

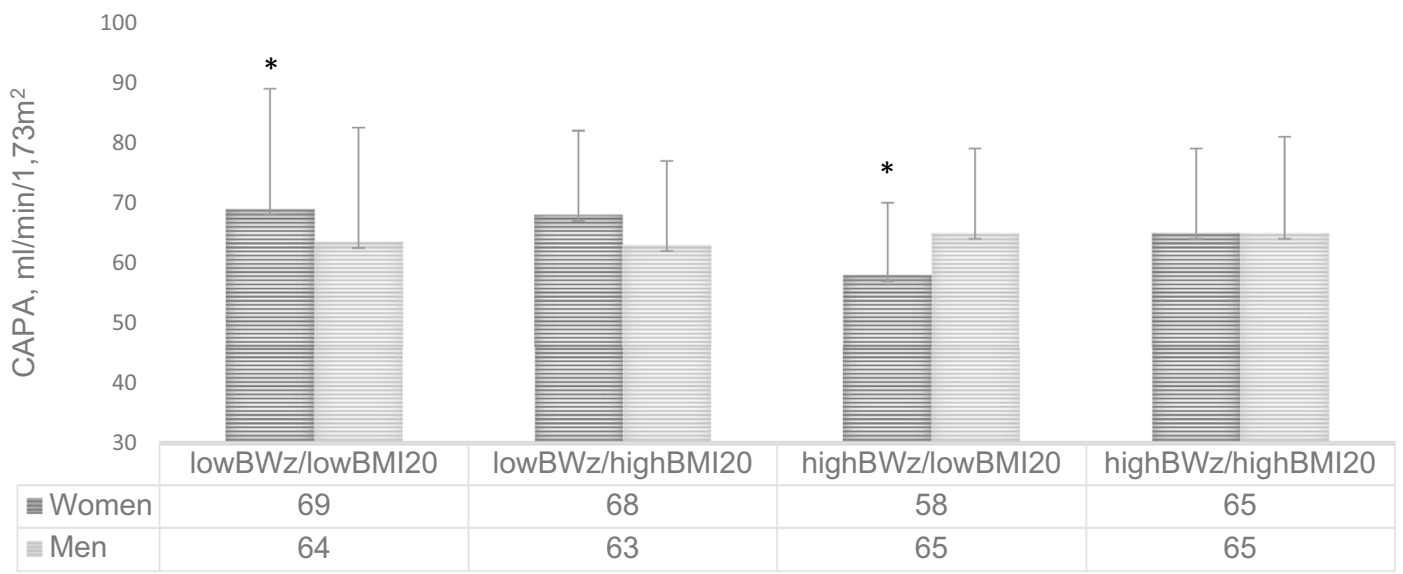

$* P=0.044$

x-axis - postnatal growth mismatch phenotype;

y- axis - eGFR measured by using CKD.EPI cystatin C (a) or CAPA (b).

a. mean $\mathrm{eGFR} \pm \mathrm{SE}$ determined by $\mathrm{CKD}$-EPI cystatin $\mathrm{C}$ equation;

b. mean eGFR $\pm \mathrm{SE}$ determined by CAPA equation.

* identifies subjects with significant difference in eGFR between growth phenotype subgroups $P<0.05$.

Fig.5 Post-natal growth patterns and adult kidney function as measured by cystatin $\mathrm{C}$ equations. a CKD-EPI cystatin C. ${ }^{*} P=0.040$. b CAPA $* P=0.044$. $x$ axis-postnatal growth mismatch phenotype; $y$ axis-eGFR measured by using CKD.EPI cystatin C (a) or CAPA

Furthermore, to the best of our knowledge we are the first to report that different postnatal growth mismatch phenotypes affect human adult kidney function. Lower cystatin C-based eGFR (both calculated using CAPA and CKD-EPI cystatin $C$ equations) was observed in adult women with high-birth weight-z-score/lowBMI20 phenotype indicating slowed postnatal growth. We could not find that in men. However, adult men with high-birth (b). ${ }^{\mathrm{a}}$ mean $\mathrm{eGFR} \pm \mathrm{SE}$ determined by CKD-EPI cystatin $\mathrm{C}$ equation; ${ }^{b}$ mean eGFR \pm SE determined by CAPA equation. *Identifies subjects with significant difference in eGFR between growth phenotype subgroups $P<0.05$

weight-z-score/highBMI20 phenotype, indicating subjects with normal to high birth weight who had higher than median BMI at 20 years of age $\left(>21.7 \mathrm{~kg} / \mathrm{m}^{2}\right)$, had a lower risk of albuminuria. These findings highlight the importance of postnatal risk factors, e.g. over-nutrition [44], for the long-term kidney outcomes. Surprisingly, subjects born with low birth weight and the higher BMI gained during the first 20 years of life (the mismatch phenotype), 
did not have lower eGFR compared to the reference group. The mismatch phenotype has been previously linked to hypertension [45] and therefore the link to kidney function was expected in our cohort.

Albuminuria, an unfavourable marker of renal outcomes [46], has been previously linked to prematurity, low birth weight and fetal growth restriction [7, 18, 47]. Our findings were in accordance with reports showing lower risk of albuminuria in adults born with normal to higher birth weight, also evident in men. A 2008 report of the National Kidney Foundation's Kidney Early Evaluation Program [48] stated that low birth weight is related to CKD explicitly in young men at 18 years of age, but the authors did not elaborate on possible mechanisms. Besides, our failure to confirm further between early life factors and UACR could be related to other unmeasured confounders such as medications, measurement errors and urine sample collection variations.

Furthermore, our findings could be explained by several possible pathways. First of all, early life factors are associated with hypertension $[12,49]$ and could thereby indirectly cause kidney damage. Therefore, we adjusted our results for PP which particularly reflects arterial stiffness, a surrogate marker for vascular risk, and target organ damage [50, 51], as well as kidney function decline [52,53]. Secondly, low nephron number at birth is assumed to directly cause premature kidney ageing and CKD [10]. Lastly, altered kidney function could be an epiphenomenon of maternal genetics for high blood pressure that has a secondary (indirect) effect on birth weight [54]. The findings of this study emphasize previous developmental research aimed at how early life factors affect future kidney damage that is essential for hypertension and ageing of the kidneys. The large reserve function of the kidneys has hampered detailed mechanistic understanding.

The strength of this study is that we report data after a long follow-up period, on average 58 years, which means current middle-aged and elderly Swedish subjects. Another strength is that we report kidney function measured by cystatin $\mathrm{C}$ and that we use different up-to-date cystatin $\mathrm{C}$ and creatinine eGFR equations against a background of different early life factors. Cystatin $\mathrm{C}$ is a more sensitive marker of kidney function compared to creatinine, as reported elsewhere [17]. It is well established that creatinine is muscle mass dependent and has lower sensitivity to detect early decreases in kidney function compared to cystatin C. Thus, our study, in contrast to studies with creatinine-based kidney function measurements, has the ability to detect associations earlier. We used both the common CKD-EPI equations, but also the LMrev and CAPA equations that were developed in the same general population from which the study cohort was retrieved in Sweden.
Limitations include that the study was restricted to people born in Sweden between 1926 and 1945, and that the study sample was relatively small. Our results could be biased due to the unequal sex distribution in the study sample, reflecting the source study (MDCS) [25], and the retrospective study design when adult participants of screening studies were back-tracked for birth data. One should not forget that there are metabolic factors, smoking, dysglycaemia, medication etc. that could have affected kidney function but were not included in this analysis that focused on early life influences.

In summary, here we report that a history of prematurity, irrespective of sex, and lower birth weight in women, are factors associated with lower cystatin C-based eGFR, while in men a history of lower birth weight (z score) is a risk marker for adult albuminuria. Cystatin C-derived eGFR equations show a higher ability to detect these findings compared to creatinine eGFR equations. A postnatal growth catch-up phenotype is not related to lower eGFR per se. The introduction of cystatin $\mathrm{C}$ in modern research has made it easier to describe these associations. We identified the protective phenotype (high-birth weight-z-score/highBMI20) for albuminuria in men and the unfavourable phenotype (high-birth weight-z-score/lowBMI20) for lower cystatin C-based eGFR in women. The observed association in men between birth weight to placenta weight and creatinine based eGFR (LMrev equation) could possibly indicate hyperfiltration and needs further investigation. The findings in general suggest that lower birth weight and postnatal growth phenotypes have a potential sex-specific influence on kidney function in middleaged and elderly Swedish subjects. Our findings suggest that women seem to be more affected by early life factors when compared to men. Further studies are warranted to address the prognostic value of early life factors for adult kidney function and risk of renal disease across the life course.

Acknowledgements To be added for funding of MDCS at baseline (see Manjer J et al. 2001).

Author contributions AL-C, AC and PMN contributed to study design. AL-C, SS, AC and PMN analysed the data. AL-C drafted the manuscript. SS, AC and PMN critically revised the manuscript. All authors read and approved the final manuscript.

Funding Open access funding provided by Lund University. Dr. Anders Christensson was supported by Grants from the Medical Faculty of Lund University, the Swedish Kidney Foundation, Njurstiftelsen, Skane University Hospital Research Fund and the Research and Development Council of Region Skane, Sweden. Cystatin C was part of a greater study and was a bonus analysis.

Data AVAILABILITY The datasets generated and/or analysed during the current study are available from the corresponding author on reasonable request. 


\section{Declarations}

Conflict of interest No potential competing interest was reported by the authors.

Ethical approval Each investigator committed to comply with legislation and to conduct the study in line with regulations, in accordance with the 1975 Declaration of Helsinki and its later amendments. The following ethical permissions apply for the cohort: MDCS (LU 51/90, 532/2006).

Open Access This article is licensed under a Creative Commons Attribution 4.0 International License, which permits use, sharing, adaptation, distribution and reproduction in any medium or format, as long as you give appropriate credit to the original author(s) and the source, provide a link to the Creative Commons licence, and indicate if changes were made. The images or other third party material in this article are included in the article's Creative Commons licence, unless indicated otherwise in a credit line to the material. If material is not included in the article's Creative Commons licence and your intended use is not permitted by statutory regulation or exceeds the permitted use, you will need to obtain permission directly from the copyright holder. To view a copy of this licence, visit http://creativecommons. org/licenses/by/4.0/.

\section{References}

1. Denic A, Glassock RJ, Rule AD (2016) Structural and functional changes with the aging kidney. Adv Chronic Kidney Dis 23(1):19-28

2. O'Sullivan ED, Hughes J, Ferenbach DA (2017) Renal aging: causes and consequences. J Am Soc Nephrol 28:407-420

3. Ferenbach DA, Bonventre JV (2015) Mechanisms of maladaptive repair after AKI leading to accelerated kidney ageing and CKD. Nat Rev Nephrol 11:264-276

4. GBD Chronic Kidney Disease Collaboration (2020) Global, regional, and national burden of chronic kidney disease, 1990 2017: a systematic analysis for the Global Burden of Disease Study 2017. Lancet 395(10225):709-733

5. Kazancioğlu R (2013) Risk factors for chronic kidney disease: an update. Kidney Int Suppl (2011). 3(4):368-371

6. Vikse BE, Irgens LM, Leivestad T, Hallan S, Iversen BM (2008) Low birth weight increases risk for end-stage renal disease. J Am Soc Nephrol 19:151-157

7. Senra JC, Carvalho MA, Rodrigues AS et al (2018) An unfavorable intrauterine environment may determine renal functional capacity in adulthood: a meta-analysis. Clinics (Sao Paulo). 73:e401

8. Eriksson JG, Salonen MK, Kajantie E, Osmond C (2018) Prenatal growth and CKD in older adults: longitudinal findings from the Helsinki birth cohort study, 1924-1944. Am J Kidney Dis 71:20-26

9. Stock K, Schmid A, Griesmaier E et al (2018) The impact of being born preterm or small for gestational age on early vascular aging in adolescents. J Pediatr 201:49-54.e1

10. Luyckx VA, Brenner BM (2015) Birth weight, malnutrition and kidney-associated outcomes-a global concern. Nat Rev Nephrol 11:135-149

11. Gonçalves GD, Walton SL, Gazzard SE et al (2020) Maternal hypoxia developmentally programs low podocyte endowment in male, but not female offspring. Anat Rec (Hoboken) 303(10):2668-2678
12. Mackenzie HS, Brenner BM (1995) Fewer nephrons at birth: a missing link in the etiology of essential hypertension? Am J Kidney Dis 26(1):91-98

13. de Boo HA, Harding JE (2006) The developmental origins of adult disease (Barker) hypothesis. Aust N Z J Obstet Gynaecol 46(1):4-14

14. Lopes van Balen VA, Spaan JJ, Cornelis T, Spaanderman MEA (2017) Prevalence of chronic kidney disease after preeclampsia. J Nephrol. 30(3):403-409

15. Cnattingius S, Villamor E, Lagerros YT, Wikström AK, Granath F (2012) High birth weight and obesity-a vicious circle across generations. Int J Obes (Lond) 36(10):1320-1324

16. Webster AC, Nagler EV, Morton RL, Masson P (2017) Chronic kidney disease. Lancet 389(10075):1238-1252

17. Dharnidharka VR, Kwon C, Stevens G (2002) Serum cystatin C is superior to serum creatinine as a marker of kidney function: a meta-analysis. Am J Kidney Dis 40(2):221-226

18. Starr MC, Hingorani SR (2018) Prematurity and future kidney health: the growing risk of chronic kidney disease. Curr Opin Pediatr 30(2):228-235

19. Manjer J, Carlsson S, Elmståhl S et al (2001) The Malmö diet and cancer study: representativity, cancer incidence and mortality in participants and non-participants. Eur J Cancer Prev 10(6):489-499

20. Hedblad B, Nilsson P, Janzon L, Berglund G (2000) Relation between insulin resistance and carotid intima-media thickness and stenosis in non-diabetic subjects. Results from a cross-sectional study in Malmö Sweden. Diabet Med. 17(4):299-307

21. Sonestedt E, Hellstrand S, Drake I et al (2016) Diet quality and change in blood lipids during 16 years of follow-up and their interaction with genetic risk for dyslipidemia. Nutrients 8(5):274

22. Gerdtsson A, Poon JB, Thorek DL et al (2015) Anthropometric measures at multiple times throughout life and prostate cancer diagnosis, metastasis, and death. Eur Urol 68(6):1076-1082

23. Lahmann PH, Gullberg B, Olsson H, Boeing H, Berglund G, Lissner L (2004) Birth weight is associated with postmenopausal breast cancer risk in Swedish women. Br J Cancer 91(9):1666-1668

24. Marsál K, Persson PH, Larsen T, Lilja H, Selbing A, Sultan B (1996) Intrauterine growth curves based on ultrasonically estimated foetal weights. Acta Paediatr 85(7):843-848

25. Berglund G, Elmstähl S, Janzon L, Larsson SA (1993) The Malmo diet and cancer study. Design and feasibility. J Intern Med 233(1):45-51

26. Pero RW, Olsson A, Berglund G, Janzon L, Larsson SA, Elmståhl S (1993) The Malmö biological bank. J Intern Med 233(1):63-67

27. Delanghe JR, Speeckaert MM (2011) Creatinine determination according to Jaffe-what does it stand for? NDT Plus 4(2):83-86

28. Björk $\mathrm{J}$ et al (2015) Accuracy of GFR estimating equations combining standardized cystatin $\mathrm{C}$ and creatinine assays: a crosssectional study in Sweden. Clin Chem Lab Med 53:403-414

29. Grubb A et al (2014) Generation of a new cystatin C-based estimating equation for glomerular filtration rate by use of 7 assays standardized to the international calibrator. Clin Chem 60:974-986

30. Inker LA et al (2012) Estimating glomerular filtration rate from serum creatinine and cystatin C. N Engl J Med 367:20-29

31. Grubb A, et al. (2014) Generation of a new cystatin C-based estimating equation for glomerular filtration rate by use of 7 assays standardized to the international calibrator. https://doi.org/10. 1373/clinchem.2013.220707.

32. Björk J, Grubb A, Larsson A et al (2015) Accuracy of GFR estimating equations combining standardized cystatin $\mathrm{C}$ and creatinine assays: a cross-sectional study in Sweden [published correction appears in Clin Chem Lab Med. 2016 May;54(5):897]. Clin Chem Lab Med. 53(3):403-414 
33. Tencer J, Thysell H, Grubb A (1996) Analysis of proteinuria: reference limits for urine excretion of albumin, protein $\mathrm{HC}$, immunoglobulin G, kappa- and lambda-immunoreactivity, orosomucoid and alpha 1-antitrypsin. Scand J Clin Lab Invest 56(8):691-700

34. Luyckx VA, Perico N, Somaschini M et al (2017) A developmental approach to the prevention of hypertension and kidney disease: a report from the low birth weight and nephron number working group. Lancet 390(10092):424-428

35. Das SK, Mannan M, Faruque AS, Ahmed T, McIntyre HD, Al MA (2016) Effect of birth weight on adulthood renal function: a bias-adjusted meta-analytic approach. Nephrology (Carlton) 21(7):547-565

36. Kistner A, Celsi G, Vanpee M, Jacobson SH (2000) Increased blood pressure but normal renal function in adult women born preterm. Pediatr Nephrol 15(3-4):215-220

37. Silverwood RJ, Pierce M, Hardy R et al (2013) Low birth weight, later renal function, and the roles of adulthood blood pressure, diabetes, and obesity in a British birth cohort. Kidney Int 84(6):1262-1270

38. Harrison SL, Mann KD, Pearce MS (2013) Early life influences kidney function at age 63-64 years, but so does adult body size: results from the newcastle thousand families birth cohort. PLoS One 8(6):e66660

39. Shehata F, Levin I, Shrim A et al (2011) Placenta/birthweight ratio and perinatal outcome: a retrospective cohort analysis. BJOG 118(6):741-747

40. Salavati N, Gordijn SJ, Sovio U et al (2018) Birth weight to placenta weight ratio and its relationship to ultrasonic measurements, maternal and neonatal morbidity: a prospective cohort study of nulliparous women. Placenta 63:45-52

41. Haavaldsen C, Samuelsen SO, Eskild A (2013) Fetal death and placental weight/birthweight ratio: a population study. Acta Obstet Gynecol Scand 92(5):583-590

42. Tomaszewski M, Charchar FJ, Maric C et al (2007) Glomerular hyperfiltration: a new marker of metabolic risk. Kidney Int 71(8):816-821

43. Palatini P (2012) Glomerular hyperfiltration: a marker of early renal damage in pre-diabetes and pre-hypertension. Nephrol Dial Transplant 27(5):1708-1714

44. Nüsken E, Voggel J, Fink G, Dötsch J, Nüsken KD (2020) Impact of early-life diet on long-term renal health. Mol Cell Pediatr $7(1): 17$
45. Sperling J, Nilsson PM (2020) Does early life programming influence arterial stiffness and central hemodynamics in adulthood? J Hypertens 38(3):481-488

46. Levey AS, Gansevoort RT, Coresh J et al (2020) Change in albuminuria and GFR as end points for clinical trials in early stages of CKD: a scientific workshop sponsored by the national kidney foundation in collaboration with the US food and drug administration and european medicines agency. Am J Kidney Dis 75(1):84-104

47. White SL, Perkovic V, Cass A et al (2009) Is low birth weight an antecedent of CKD in later life? A systematic review of observational studies. Am J Kidney Dis 54(2):248-261

48. Li S, Chen SC, Shlipak M et al (2008) Low birth weight is associated with chronic kidney disease only in men. Kidney Int 73(5):637-642

49. Knop MR, Geng TT, Gorny AW et al (2018) Birth weight and risk of type 2 diabetes mellitus, cardiovascular disease, and hypertension in adults: a meta-analysis of 7646267 participants from 135 studies. J Am Heart Assoc 7(23):e008870

50. O'Rourke MF, Safar ME (2005) Relationship between aortic stiffening and microvascular disease in brain and kidney: cause and logic of therapy. Hypertension 46(1):200-204

51. Vasan RS, Short MI, Niiranen TJ et al (2019) Interrelations between arterial stiffness, target organ damage, and cardiovascular disease outcomes. J Am Heart Assoc. 8(14):e012141

52. Safar ME, Plante GE, Mimran A (2015) Arterial stiffness, pulse pressure, and the kidney. Am J Hypertens 28(5):561-569

53. Geng TT, Talaei M, Jafar TH, Yuan JM, Koh WP (2019) Pulse pressure and the risk of end-stage renal disease among Chinese adults in Singapore: the Singapore Chinese health study. J Am Heart Assoc. 8(23):e013282

54. Warrington NM, Beaumont RN, Horikoshi M et al (2019) Maternal and fetal genetic effects on birth weight and their relevance to cardio-metabolic risk factors. Nat Genet 51(5):804-814

Publisher's Note Springer Nature remains neutral with regard to jurisdictional claims in published maps and institutional affiliations. 